ARTICLE

DOI: $10.1038 /$ s41467-018-03765-0

OPEN

\title{
Tuning defects in oxides at room temperature by lithium reduction
}

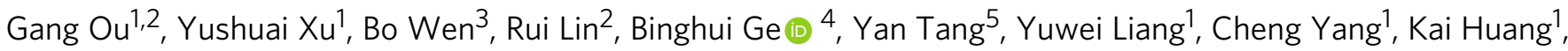

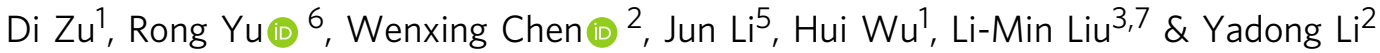

Defects can greatly influence the properties of oxide materials; however, facile defect engineering of oxides at room temperature remains challenging. The generation of defects in oxides is difficult to control by conventional chemical reduction methods that usually require high temperatures and are time consuming. Here, we develop a facile room-temperature lithium reduction strategy to implant defects into a series of oxide nanoparticles including titanium dioxide $\left(\mathrm{TiO}_{2}\right)$, zinc oxide $(\mathrm{ZnO})$, tin dioxide $\left(\mathrm{SnO}_{2}\right)$, and cerium dioxide $\left(\mathrm{CeO}_{2}\right)$. Our lithium reduction strategy shows advantages including all-room-temperature processing, controllability, time efficiency, versatility and scalability. As a potential application, the photocatalytic hydrogen evolution performance of defective $\mathrm{TiO}_{2}$ is examined. The hydrogen evolution rate increases up to $41.8 \mathrm{mmol} \mathrm{g}^{-1} \mathrm{~h}^{-1}$ under one solar light irradiation, which is $\sim 3$ times higher than that of the pristine nanoparticles. The strategy of tuning defect oxides used in this work may be beneficial for many other related applications.

\footnotetext{
${ }^{1}$ State Key Laboratory of New Ceramics and Fine Processing, School of Materials Science and Engineering Tsinghua University, 100084 Beijing, China. ${ }^{2}$ Department of Chemistry and Collaborative Innovation Center for Nanomaterial Science and Engineering, Tsinghua University, 100084 Beijing, China. ${ }^{3}$ Beijing Computational Science Research Center, 100193 Beijing China. ${ }^{4}$ Beijing National Laboratory for Condensed Matter Physics, Institute of Physics, Chinese Academy of Science, 100190 Beijing, China. ${ }^{5}$ Department of Chemistry and Key Laboratory of Organic Optoelectronics and Molecular Engineering of Ministry of Education, Tsinghua University, 100084 Beijing, China. ${ }^{6}$ National Center for Electron Microscopy in Beijing, School of Materials Science and Engineering, Tsinghua University, 100084 Beijing, China. ${ }^{7}$ School of Physics, Beihang University, 100191 Beijing, China. These authors contributed equally: Gang Ou, Yushuai Xu, Bo Wen. Correspondence and requests for materials should be addressed to H.W. (email: huiwu@tsinghua.edu.cn) or to L.-M.L. (email: liminliu@buaa.edu.cn) or to Y.L. (email: ydli@tsinghua.edu.cn)
} 
D efect engineering of oxide materials has been a major focus in materials science, since the fundamental physical and chemical properties of oxide materials greatly rely on their defect structures ${ }^{1-7}$. For example, the optical absorption of oxides can be greatly enhanced by implanting defects ${ }^{8,9}$. Besides, defects have also been reported to enhance catalytic activity by taking advantage of defect sites as active centers ${ }^{10-12}$. Therefore, the science and technology behind the tuning of defect structures in oxide materials have attracted increasing attention in the past several decades ${ }^{13-17}$. Recently, $\mathrm{Li}$ et al. reported a chemical leaching method to prepare jagged nanowires with defective sites at the surface. In their work, they found highly enhanced oxygen reduction reaction performance ${ }^{12}$. Chen et al. reported defective "black" $\mathrm{TiO}_{2}$ with significantly enhanced photocatalytic activity by hydrogenating pristine $\mathrm{TiO}_{2}$ nanoparticles in a 20 bar $\mathrm{H}_{2}$ atmosphere at $200{ }^{\circ} \mathrm{C}$ for 5 days ${ }^{18}$. Reductive metals have also been introduced to tune the defects in $\mathrm{TiO}_{2}$ at high temperatures, such as $\mathrm{Mg}$ and $\mathrm{Al}^{19,20}$. In addition, Nakajima et al. reported defective $\mathrm{TiO}_{2}$ by high energy laser irradiation, providing another strategy to tune the defects in oxide materials ${ }^{21,22}$. Nevertheless, controllable defect engineering in oxide materials under ambient conditions remains challenging. Meanwhile, defect engineering has emerged as an effective strategy to tune the electronic structure of metal oxides, which is vital for many applications $s^{2,5,23-26}$.

According to Ellingham diagrams ${ }^{27}$, lithium metal, which possesses high reductive activity, can potentially reduce a significant number of metal oxides at room temperature. Therefore, Li reduction may provide exciting opportunities to partially reduce a series of oxide materials at room temperature, removing oxygen, generating oxygen vacancies and related defects in these materials.

Here, we show that by simply grinding pristine oxide powders with lithium metal powder, followed by an acid leaching process for the removal of lithium oxides from the mixture, a series of oxide materials including $\mathrm{TiO}_{2}, \mathrm{ZnO}, \mathrm{SnO}_{2}$, and $\mathrm{CeO}_{2}$ are implanted with a high concentration of defects. Moreover, we demonstrate that the $\mathrm{TiO}_{2}$ nanoparticles with tunable defects demonstrate high activity and long stability as a photocatalyst for the hydrogen evolution reaction.

\section{Results}

Thermodynamic calculations. To prove that lithium can effectively reduce $\mathrm{TiO}_{2}, \mathrm{SnO}_{2}, \mathrm{ZnO}$ etc., as represented in Eq. (1), the reaction enthalpies were calculated. As shown in Supplementary Table 1, the enthalpy changes of reaction represented by Eq. (1) are all negative, which means the reactions are exothermic. Furthermore, for example, $\Delta G$ for the reaction $4 \mathrm{Li}+\mathrm{TiO}_{2} \rightarrow \mathrm{Ti}+$ $2 \mathrm{Li}_{2} \mathrm{O}$ is $-233.6 \mathrm{~kJ} \mathrm{~mol}^{-1}$ at $298 \mathrm{~K}$, indicating the reaction can occur spontaneously at room temperature.

$$
\mathrm{MO}_{y}+2 x \mathrm{Li} \rightarrow \mathrm{MO}_{y-x}+x \mathrm{Li}_{2} \mathrm{O} .
$$

Synthesis and characterization of defective oxides. We applied the lithium reduction method to create defects in $\mathrm{TiO}_{2}$ nanoparticles (P25, Degussa, commercially available) since $\mathrm{TiO}_{2}$ has been widely studied as a high performance photocatalyst in recent decades ${ }^{28,29}$. $\mathrm{TiO}_{2}$ and lithium powders were weighed and placed in a mortar, followed by the addition of dimethyl carbonate (DMC) as a dispersant. The mixtures were ground up and then removed by dissolving the generated lithium oxide with dilute hydrochloric acid $(\mathrm{HCl})$. The detailed process is shown in Supplementary Fig. 1. After centrifuging and washing, the obtained powders were dried for characterization. As shown in Fig. 1a, the lithium-reduced $\mathrm{TiO}_{2}$ samples appear in different colors ranging from blue to black, in marked contrast to the white pristine $\mathrm{TiO}_{2}$ powders. The color change of $\mathrm{TiO}_{2}$ may be ascribed to the enhanced absorption of visible light and thus we named them "Black" $\mathrm{TiO}_{2}$. Figure $1 \mathrm{~b}$ shows the identical crystalline structure with the anatase phase in majority and that of rutile in minority for various $\mathrm{TiO}_{2}$ samples, which demonstrates that our lithium reduction method does not alter the intrinsic crystal structure of the $\mathrm{TiO}_{2}$ nanoparticles. As shown in the Raman spectra in Supplementary Fig. 2, the Raman vibration band of pristine $\mathrm{TiO}_{2}$ powders exhibits an identical configuration to that of the lithiumreduced $\mathrm{TiO}_{2}$ except for the broadening of the $E_{\mathrm{g}}$ peak at $\sim 148 \mathrm{~cm}$ ${ }^{-1}$ (Supplementary Fig. 3). It is found that the particle size of lithium-reduced $\mathrm{TiO}_{2}$ is almost the same as that of pristine $\mathrm{TiO}_{2}$, based on the morphology characterized by scanning electron microscopy (SEM, Supplementary Fig. 4), which indicates that the room-temperature conditions do not cause further growth of the grains. To demonstrate that our approach can be used as a versatile method to generate defects in other oxides, we prepared similar "Black" $\mathrm{ZnO}, \mathrm{SnO}_{2}$, and $\mathrm{CeO}_{2}$ nanoparticles by the lithium reduction route (Fig. 1c) without changing their crystal structures (Fig. 1d-f) or particle size (Supplementary Fig. 5).

To evaluate the defects of lithium-reduced $\mathrm{TiO}_{2}, \mathrm{X}$-ray photoelectron spectroscopy (XPS) of elemental $\mathrm{O}$ and $\mathrm{Ti}$ are provided. Three typical XPS peaks at $\sim 530.6,531.9$, and $532.8 \mathrm{eV}$ (labeled as O1, O2, and O3) in Fig. 2a can be attributed to lattice oxygen, oxygen defects, and surface-adsorbed oxygen species, respectively ${ }^{18,30}$. As observed in Fig. 2a, the content of oxygen defects (O2) in lithium-reduced $\mathrm{TiO}_{2}$ exhibits a significant increase compared with that of pristine $\mathrm{TiO}_{2}$, suggesting that the active lithium can efficiently remove partial oxygen elements and then generate more oxygen vacancies in the lattice of $\mathrm{TiO}_{2}$ powders. In addition, the content of oxygen defects in lithiumreduced $\mathrm{TiO}_{2}$ powders increased noticeably with increasing lithium metal powders, which indicates the facile use of lithium to tune the defect content in $\mathrm{TiO}_{2}$ powder. Furthermore, the $\mathrm{Ti}$ $2 \mathrm{p}$ spectra of $\mathrm{TiO}_{2}$ powder also demonstrates that infrequent $\mathrm{Ti}^{3+}$ was produced from the generation of oxygen vacancies ${ }^{30}$ (Fig. $2 \mathrm{~b}$ and Supplementary Fig. 6). Since XPS analysis is rather insensitive to the detection of $\mathrm{Ti}^{3+}$, we applied electron paramagnetic resonance (EPR) measurements to study the titanium defects in $\mathrm{TiO}_{2}$ powders. The indicator values of $\mathrm{g}$ peaks at 1.973 and 2.002 correspond to $\mathrm{Ti}^{3+}$ and oxygen vacancies in the lattice, respectively ${ }^{30,31}$ (Fig. 2c). Consistent with the results of the XPS spectra, the content of $\mathrm{Ti}^{3+}$ and oxygen vacancies in lithium-reduced $\mathrm{TiO}_{2}$ are significantly higher than those in the pristine powder. It is important to note that no elemental lithium was found in either the pristine $\mathrm{TiO}_{2}$ or the lithium-reduced $\mathrm{TiO}_{2}$ powders as determined by $\mathrm{Li}$ 1s spectra ${ }^{32}$ (Supplementary Fig. 7), which suggests that lithium oxides can be thoroughly removed by acid treatment. Further characterization by transmission electron microscopy (TEM) in Fig. $2 \mathrm{~d}$ and e reveals that the pristine $\mathrm{TiO}_{2}$ has no disordered domains, but after treatment with $5 \% \mathrm{Li}$ a disordered layer with a thickness of $2 \mathrm{~nm}$ was observed. We attribute this to the deprivation of surface lattice oxygen by elemental lithium during the contact reaction process. Interestingly, we also found that the specific surface area of $\mathrm{TiO}_{2}$ nanoparticles increased from 49.88 to $55.28 \mathrm{~m}^{2} \mathrm{~g}^{-1}$ after lithium reduction treatment, which can be attributed to the generated defective surface structure.

Moreover, we investigated the conductivity of the $\mathrm{TiO}_{2}$ nanoparticles (Supplementary Fig. 8), obtaining significantly enhanced conductivity after lithium reduction treatment due to the generated disordered surface layer. It is well-known that the existence of the $\mathrm{Ti}^{3+}$ can enhance the conductivity of $\mathrm{TiO}_{2}{ }^{33-35}$. As shown in Supplementary Fig. 9, the existence of oxygen vacancies produces excess electrons, which transforms $\mathrm{Ti}^{4+}$ into 
a

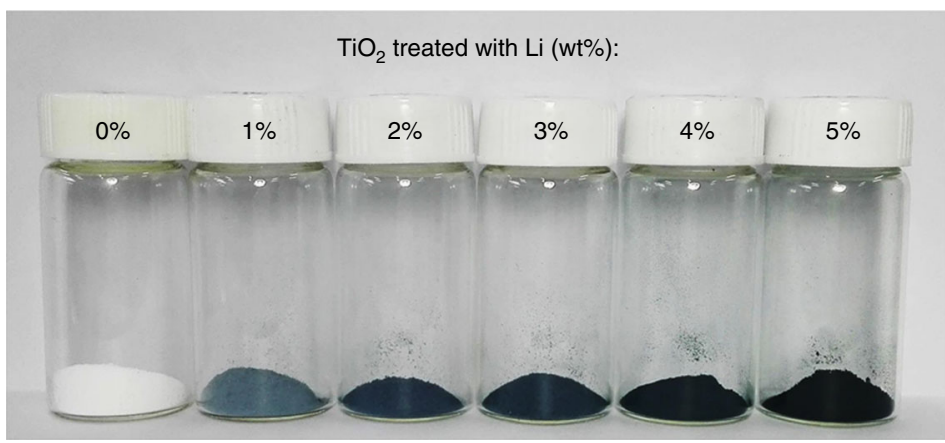

b

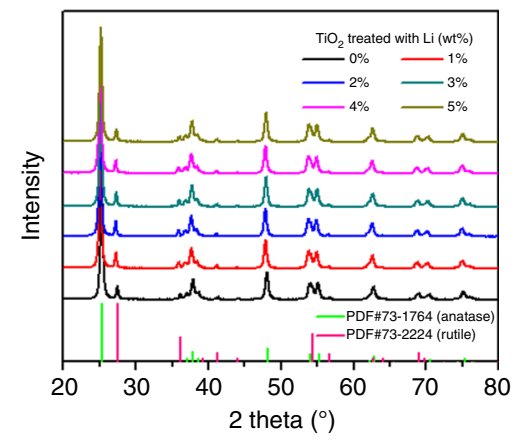

C

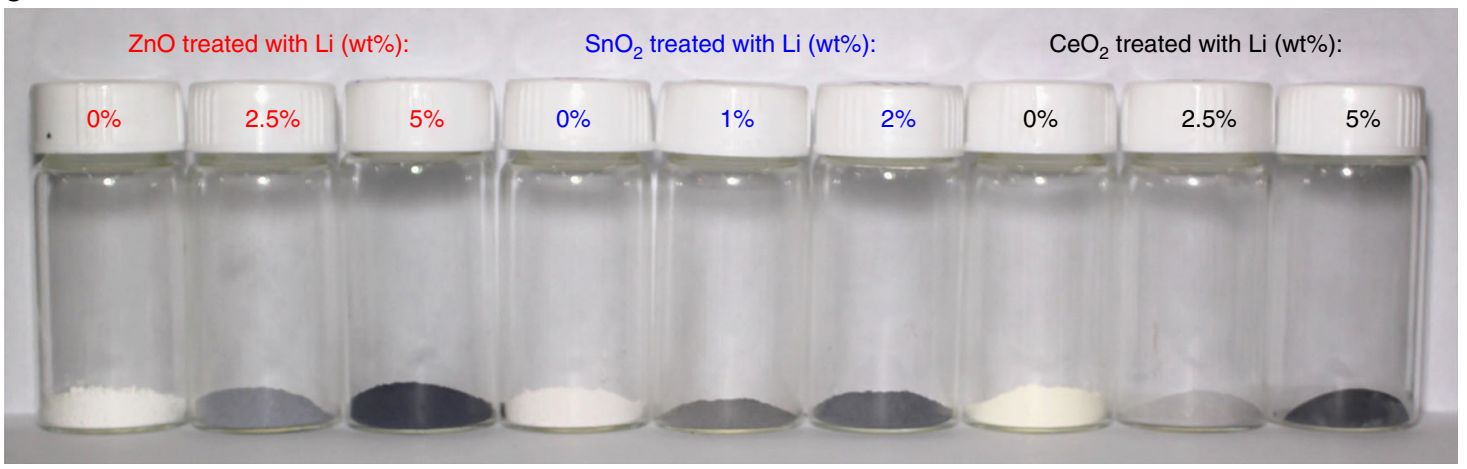

d

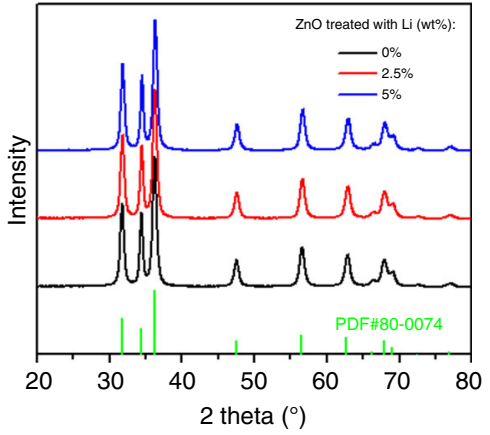

e

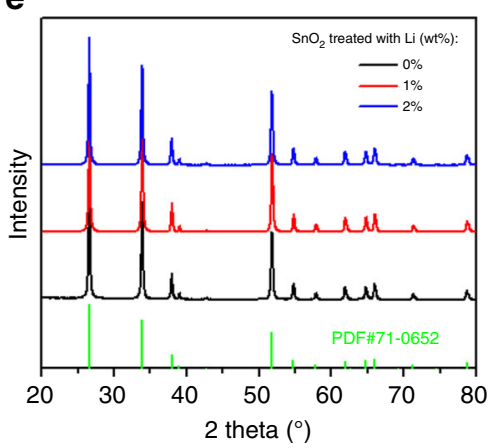

f

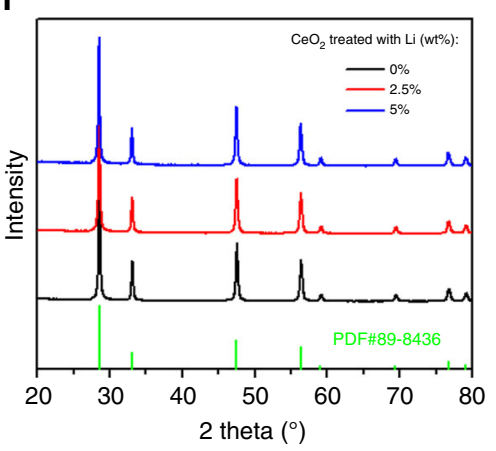

Fig. 1 Pristine and lithium-reduced oxide nanoparticles. a Photograph of $\mathrm{TiO}_{2}$. b XRD patterns of $\mathrm{TiO}_{2}$. c Photograph of $\mathrm{ZnO}_{2} \mathrm{SnO}_{2}$, and $\mathrm{CeO}$, respectively. d-f XRD patterns of $\mathrm{ZnO}, \mathrm{SnO}_{2}$, and $\mathrm{CeO}_{2}$, respectively

$\mathrm{Ti}^{3+}$. Meanwhile, the concentration of $\mathrm{Ti}^{3+}$ greatly depends on the amount of oxygen vacancies $\left(\mathrm{O}_{\mathrm{v}}\right)$. The excess electrons can freely hop at room temperature ${ }^{36-38}$, which is likely the main reason for the high conductivity of the reduced $\mathrm{TiO}_{2}$. Beyond $\mathrm{TiO}_{2}$, the "Black" $\mathrm{ZnO}, \mathrm{SnO}_{2}$, and $\mathrm{CeO}_{2}$ exhibit similar defects structure compared with $\mathrm{TiO}_{2}$. We observed that the content of oxygen defects in all the oxides significantly increased after lithium reduction treatment (Supplementary Fig. 10), indicating that the oxygen defects have been successfully implanted in the oxides. At the same time, the binding energy of the metal cations in the oxides slightly decreased after lithium reduction treatment due to the implanted defects (Supplementary Fig. 11). In addition, no peaks related to $\mathrm{Li}$ were detected in the lithium-reduced oxides $^{32}$ (Supplementary Fig. 12), which further confirms that all the remaining lithium oxides were fully removed during the acid leaching process.

In order to investigate the effect of $\mathrm{Li}$ metal powder particle size on the reduction of the oxides, we prepared defective $\mathrm{TiO}_{2}$ nano-powders reduced by $5 \mathrm{wt} \% \mathrm{Li}$ powder and a single piece of Li. Here, the average diameter of the Li powder particles was $\sim 30$ $\mu \mathrm{m}$ (Supplementary Fig. 13). As shown in Supplementary Fig. 14, it is apparent that the defective $\mathrm{TiO}_{2}$ powders produced with the $\mathrm{Li}$ powder and the $\mathrm{Li}$ piece exhibit the same color depth, indicating their defective content to be consistent. In addition, we also compared different content of DMC on the reduction of $\mathrm{TiO}_{2}$ nano-powder. As shown in Supplementary Fig. 15, it can be seen that the color of the defective $\mathrm{TiO}_{2}$ did not change with the dispersant content from 1:10, 1:20 to 1:30. Our first-principles calculations show that the formation energy of an oxygen vacancy $\left(\mathrm{O}_{\mathrm{v}}\right)$ in $\mathrm{TiO}_{2}$ is $3.77 \mathrm{eV}$ per oxygen, while the corresponding formation energy of losing one oxygen atom in DMC is $4.80 \mathrm{eV}$ per oxygen, which is $1.03 \mathrm{eV}$ larger than the corresponding formation energy through capturing oxygen from $\mathrm{TiO}_{2}$. Thus, $\mathrm{Li}$ prefers to capture oxygen from $\mathrm{TiO}_{2}$ instead of DMC.

From the above results, we are confident that uniform defects were successfully implanted at the surface of the oxide nanopowders by our lithium reduction strategy, which can be ascribed to several reasons. Firstly, the lithium metal can react with oxides at room temperature according to the Ellingham diagrams and our calculated results. Thus the lithium metal can draw $\mathrm{O}$ from the oxides to generate $\mathrm{Li}_{2} \mathrm{O}$ as well as defective oxides during the grinding process. Secondly, the lithium can be continuously 
a

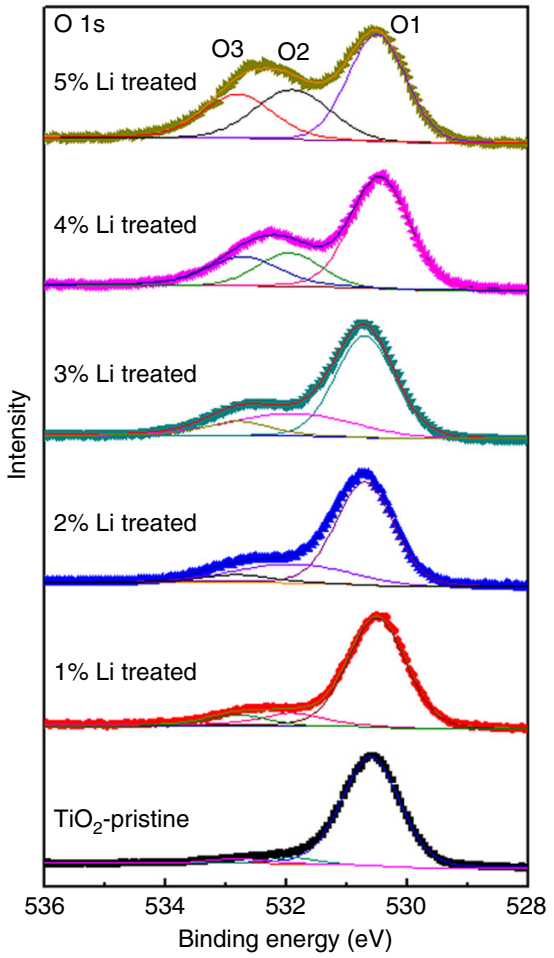

d

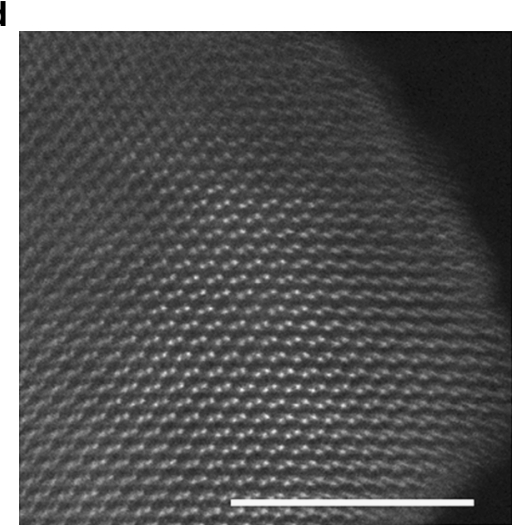

b
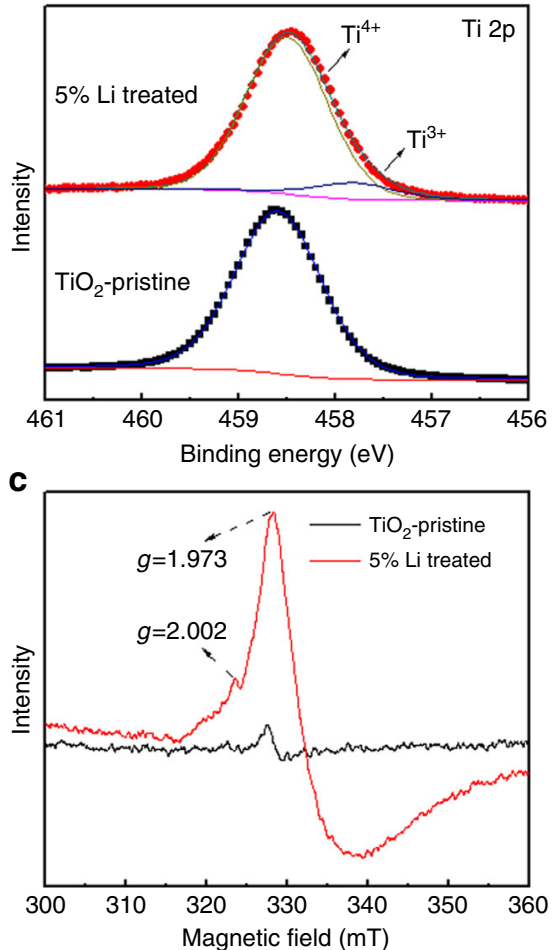

e

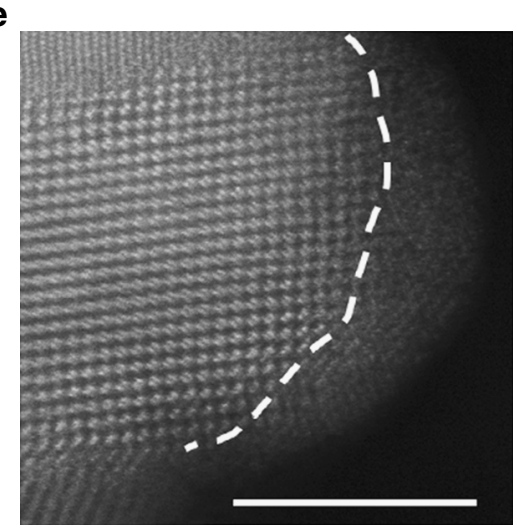

Fig. 2 Defect characterization of pristine and lithium-reduced $\mathrm{TiO}_{2}$ nanoparticles. a, b XPS spectra. c EPR spectra. d, e High angle annular dark field (HAADF) images of pristine and $5 \%$ Li-treated $\mathrm{TiO}_{2}$. Scale bars, $5 \mathrm{~nm}$

extended during the grinding process because of its high ductility, resulting in the constant exposure of fresh lithium to react with oxides, until the lithium metal fully reacts. Lastly, the lithium powders and oxide nano-powders can be uniformly mixed with the addition of DMC, which is beneficial for the uniform implantation of defects at the surface of the oxide nano-powders during the grinding process. In addition, the reaction between $\mathrm{Li}$ and $\mathrm{TiO}_{2}$ occurs at the interface, which captures the oxygen atoms at the $\mathrm{TiO}_{2}$ surface. Although the oxygen vacancies are initially generated at the surface, they prefer to diffuse into the subsurface or the inner layer (Supplementary Fig. 16). The subsurface $\mathrm{O}_{\mathrm{v}}$ is more stable by $\sim 0.13 \mathrm{eV}$ than the one at the surface, as suggested in previous experiments and theory ${ }^{39-41}$. The calculated diffusion energy barrier is $\sim 0.7 \mathrm{eV}$ (Supplementary Fig. 16), which indicates that the $\mathrm{O}_{\mathrm{v}}$ has the tendency to diffuse from the surface to the inner layer region at room temperature.

As discussed above, the defective oxides often exhibit superior properties than their pristine counterparts for a range of applications ${ }^{2,5,23-26}$. Therefore we expect the defective metal oxides fabricated in this work to have many potential applications. In the following, as examples of potential applications, we examined the photocatalytic degradation of organic pollutants and the hydrogen evolution of our defective $\mathrm{TiO}_{2}$ nano-powder.

Photocatalytic properties of defective $\mathrm{TiO}_{2}$ nano-powder. Supplementary Fig. 17 shows the UV-Vis-NIR absorption spectra of $\mathrm{TiO}_{2}$ powders at different reduction levels to explain the optical absorption change induced by the formation of defects. It is found that the absorption of visible and near-infrared light for lithium-reduced $\mathrm{TiO}_{2}$ was enhanced significantly compared to that of pristine $\mathrm{TiO}_{2}$ powder, and there is a positive correlation between the enhancement and the lithium metal content. According to the fitted optical bandgap, as given by the Tauc equation ${ }^{42}$, the bandgap of the $\mathrm{TiO}_{2}$ narrowed from 3.3 to $3.1 \mathrm{eV}$ after treatment with lithium (Supplementary Fig. 18), suggesting that the introduction of defects in reduced $\mathrm{TiO}_{2}$ can slightly narrow the bandgap and also widen the range of light absorption. 
a

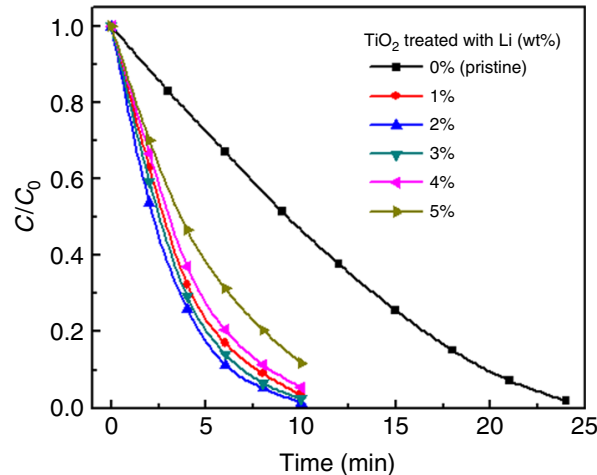

C

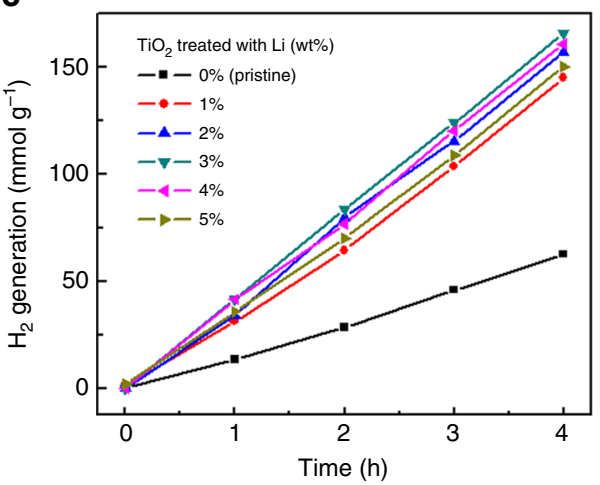

b

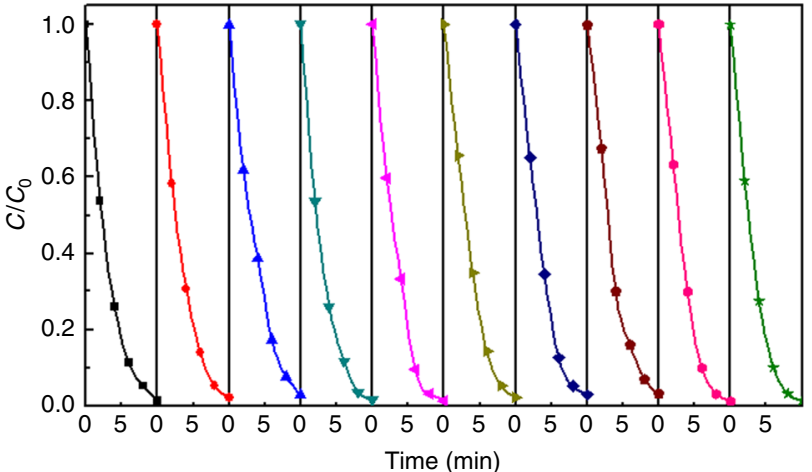

d

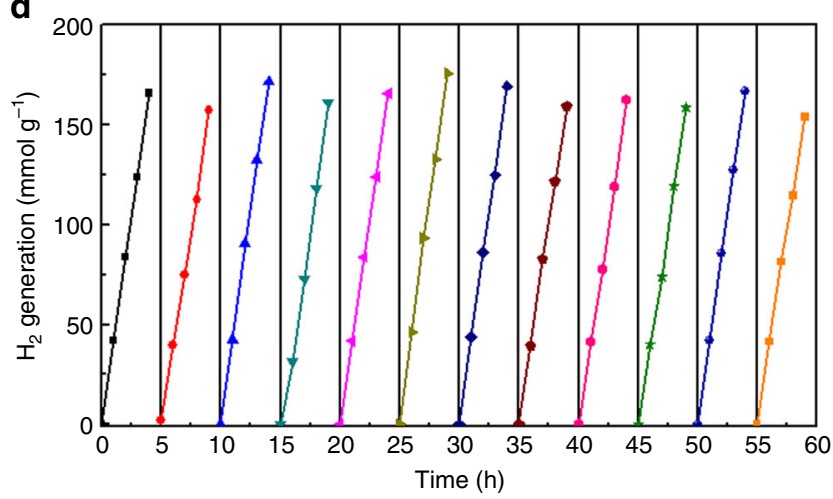

Fig. 3 Photocatalytic properties of pristine and lithium-reduced $\mathrm{TiO}_{2}$ nanoparticles. a, b Photocatalytic activity and stability for degradation of RhB. c, d Photocatalytic activity and stability for hydrogen evolution

In addition to optical absorption properties, the separation and migration of non-equilibrium state carriers are also affected by the defect formation. The fluorescence in solid steady state could shed light on the transportation and recombination kinetics of photogenerated electron-hole pairs qualitatively. Supplementary Fig. 19 displays that both the pristine and defective $\mathrm{TiO}_{2}$ show their emission peaks at $412 \mathrm{~nm}$ when excited by the $330 \mathrm{~nm}$ light. The lower fluorescence emission intensity of defective $\mathrm{TiO}_{2}$ implies that its recombination of excited state electron-hole pairs is less severe than that of pristine $\mathrm{TiO}_{2}$, which may endow defective $\mathrm{TiO}_{2}$ with a longer lifetime of carriers. Transient state fluorescence tests confirm our prediction successfully (Supplementary Fig. 20). The fluorescence decay profile showed that the lifetime of photogenerated carriers in defective $\mathrm{TiO}_{2}(280 \mathrm{ps})$ is longer than that of pristine $\mathrm{TiO}_{2}(230 \mathrm{ps})$, which proves that the defects promote the separation of photogenerated carriers. Furthermore, the optical absorption of $\mathrm{ZnO}, \mathrm{SnO}_{2}$, and $\mathrm{CeO}_{2}$ nanoparticles was also measured, showing greatly enhanced absorption (Supplementary Fig. 21) and a marginally narrowed bandgap (Supplementary Fig. 22) after the lithium reduction treatment-induced defects implantation in these oxides.

Theoretically, the defective $\mathrm{TiO}_{2}$ photocatalyst with wider solar spectrum response range and longer charge carrier lifetime will exhibit higher activity. In line with our deduction, we observed a significant improvement of rhodamine $\mathrm{B}(\mathrm{RhB})$ photocatalytic degradation efficiency in lithium-reduced $\mathrm{TiO}_{2}$ compared with that in pristine $\mathrm{TiO}_{2}$ (Fig. 3a). The time taken for $\mathrm{TiO}_{2}$ photocatalyst to decompose $\mathrm{RhB}$ under solar light decreased from 24 to $10 \mathrm{~min}$ after the lithium reduction treatment. Interestingly, the photocatalytic degradation activity demonstrated first an increase and then a decrease with increasing lithium content, and attained optimization after treatment with $2 \% \mathrm{Li}$. Therefore, we repeated the degradation of $\mathrm{RhB}$ nine times based on the $2 \%$ Li-reduced $\mathrm{TiO}_{2}$ nanoparticles (Fig. 3b). We clearly observed that the lithium-reduced $\mathrm{TiO}_{2}$ nanoparticles remain constant after nine cycles' degradation of $\mathrm{RhB}$, suggesting their excellent stability. Afterwards, we tested the photocatalytic hydrogen evolution activity. Compared with pristine $\mathrm{TiO}_{2}$, the lithium-reduced $\mathrm{TiO}_{2}$ revealed significantly enhanced photocatalytic activity (Fig. 3c). The photocatalytic hydrogen evolution rate increased from $13.4 \mathrm{mmol} \mathrm{g}^{-1} \mathrm{~h}^{-1}$ (pristine $\mathrm{TiO}_{2}$ ) to $41.8 \mathrm{mmol} \mathrm{g}^{-1} \mathrm{~h}^{-1}$ (3\% Li-reduced $\left.\mathrm{TiO}_{2}\right)$ under a full solar light (containing ultraviolet, visible and near-infrared light at an irradiation density of $100 \mathrm{~mW} \mathrm{~cm}^{-2}$ ). In addition, the photocatalytic hydrogen evolution activity also demonstrated an initial increase and then decrease with increasing lithium content, and achieved its peak after treatment with $\sim 3 \% \mathrm{Li}$, which is consistent with the above photocatalytic decomposition activity. We noticed that the defects have various impacts on the photocatalytic property; that is, the photocatalytic property is proportional to the content of defects up to a certain point, due to the enhanced light absorption and active sites. However, excess defects are detrimental to the photocatalytic activity since they can also act as charge recombination centers ${ }^{31,43}$. Subsequently, we repeated the hydrogen evolution experiments 11 times based on the $3 \% \mathrm{Li}$-treated $\mathrm{TiO}_{2}$ nanoparticles (Fig. 3d). It is apparent that the lithium-reduced $\mathrm{TiO}_{2}$ demonstrates consistently excellent activity after long-term cycles, which also indicates the superior stability of the defective $\mathrm{TiO}_{2}$ and the implanted defects within them. Based on the above photocatalytic results, we suggest that the enhanced photocatalytic properties can be attributed to the implanted defects in the $\mathrm{TiO}_{2}$ nanoparticles. In order to prove this, we further characterized the photocatalytic degradation activity of lithium-reduced $\mathrm{TiO}_{2}$ nanoparticles after annealing at $400{ }^{\circ} \mathrm{C}$ for $1 \mathrm{~h}$ to remove the generated defects (Supplementary Fig. 23). The results showed that the photocatalytic performance 


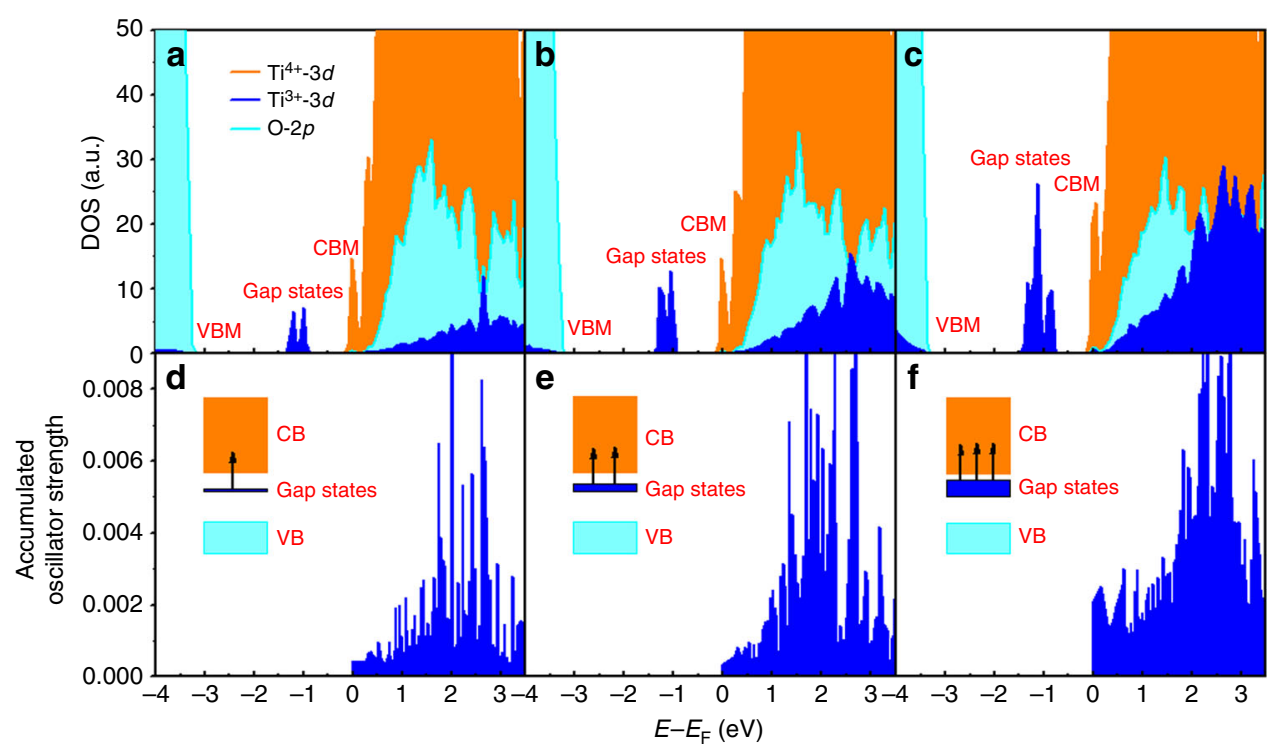

Fig. 4 Density of states (DOS) and corresponding accumulated oscillator strength of defective anatase $\mathrm{TiO}_{2}$. a-c $\mathrm{DOS}$ of $\mathrm{TiO}_{2}$ surface with $1 \mathrm{O}_{\mathrm{v}}, 2 \mathrm{O}_{\mathrm{v}}$, and $4 \mathrm{O}_{v}$ per slab, respectively. d-f Oscillator strength of $\mathrm{TiO}_{2}$ surface with $1 \mathrm{O}_{v}, 2 \mathrm{O}_{v}$, and $4 \mathrm{O}_{v}$ per slab, respectively. The oscillator strength was calculated for transitions from gap states to conduction band $(\mathrm{CB})$. The orange, blue, and light green area in the DOS stand for projected $\mathrm{DOS}$ of $\mathrm{Ti}^{4+}$ ions, $\mathrm{Ti}^{3+}$ ions, and $\mathrm{O}^{2-}$ ions, respectively. The gap state, valence band maximum (VBM), and conduction band minimum (CBM) are indicated in the DOS. Accumulated oscillator strength is calculated by summing the component data of $y$ and $z$ direction in Supplementary Fig. 24

of defective $\mathrm{TiO}_{2}$ significantly declined after annealing. Furthermore, we also compared the photocatalytic hydrogen evolution activity of the lithium-reduced $\mathrm{TiO}_{2}$ nanoparticles with previously reported works (Supplementary Table 2), which indicates that our defective $\mathrm{TiO}_{2}$ by lithium reduction possesses superior photocatalytic activity.

Theoretical calculations. To understand the origin of the change in electronic and optical properties of lithium-reduced $\mathrm{TiO}_{2}$ nanoparticles, the electronic structure was predicted by firstprinciples calculations. Firstly, the density of states (DOS) for reduced $\mathrm{TiO}_{2}$ with different $\mathrm{O}_{\mathrm{v}}$ concentrations was examined (Fig. $4 \mathrm{a}-\mathrm{c}$ ). As indicated in Fig. 4, the valence band maximum (VBM) was mainly composed of O- $2 p$ orbitals, and the conduction band minimum (CBM) mainly consisted of Ti-3d orbitals. Interestingly, the change of $\mathrm{O}_{\mathrm{v}}$ concentrations $\left(1 \mathrm{O}_{\mathrm{v}}, 2 \mathrm{O}_{\mathrm{v}}\right.$, and $4 \mathrm{O}_{\mathrm{v}}$ ) does not affect the positions of either VBM or CBM, so the intrinsic bandgap is not affected by $\mathrm{O}_{\mathrm{v}}$. As found above, the intrinsic band gap of $\mathrm{TiO}_{2}$ is only changed from 3.3 to $3.1 \mathrm{eV}$ after inducing $\mathrm{O}_{v}$, which is consistent with the theoretical results.

Considering that $\mathrm{O}_{\mathrm{v}}$ does not affect the intrinsic bandgap, it is natural to argue why the light absorption of $\mathrm{TiO}_{2}$ containing $\mathrm{O}_{\mathrm{v}}$ is greatly enhanced compared with that of pristine $\mathrm{TiO}_{2}$. It has been previously reported that excess electrons can be introduced by $\mathrm{O}_{\mathrm{v}}{ }^{44}$. Meanwhile, excess electrons can be easily trapped by the $\mathrm{Ti}^{4}$ + ions to form $\mathrm{Ti}^{3+}$ ions, and the gap states induced by $\mathrm{Ti}^{3+}$ ions exist within the intrinsic band gap ${ }^{45}$. The projected DOS of $\mathrm{Ti}^{4+}$, $\mathrm{Ti}^{3+}$ and $\mathrm{O}^{2-}$ ions are shown in Fig. $4 \mathrm{a}-\mathrm{c}$, respectively. The orange, blue, and light green areas represent the corresponding projected DOS of $\mathrm{Ti}^{4+}$ ions, $\mathrm{Ti}^{3+}$ ions, and $\mathrm{O}^{2-}$ ions, respectively. As shown in Fig. $4 \mathrm{a}-\mathrm{c}$, the peak of gap states (the blue peaks) gradually become larger and broadened with the increase of $\mathrm{O}_{\mathrm{v}}$ concentration. Meanwhile, the intensity of PDOS of $\mathrm{Ti}^{3+}$ ions in the $\mathrm{CB}$ region, especially the peak $\sim 2 \mathrm{eV}$ above $\mathrm{CBM}$ was also greatly enhanced ${ }^{46}$. In fact, the CBM of $\mathrm{TiO}_{2}$ is composed of empty $t_{2 g}$ orbitals, so when the excess electrons exist in the $\mathrm{TiO}_{2}$ system, each of them will firstly fill one of the three nearly degenerated $t_{2 g}$ orbitals and leaves two unoccupied states.
The filled $t_{2 g}$ orbitals become the gap states, while the other two unoccupied orbitals of $t_{2 g}$ move to higher energy levels, corresponding to the resonance of the gap states, consistent with the Jahn-Teller effect ${ }^{46}$.

In order to know whether the gap states affect photoabsorption, the oscillator strength of transitions from gap states to the $\mathrm{CB}$ were further calculated for $\mathrm{Ti}^{3+}$. As shown in Fig. $4 \mathrm{~d}-\mathrm{f}$, some peaks at $\sim 2 \mathrm{eV}$ appear for the oscillator strength of the transition from gap states to the CB. Such results clearly suggest that the gap states induced by $\mathrm{O}_{\mathrm{v}}$ can enhance the photoadsorption through $d$ $-d$ transitions between $\mathrm{Ti}^{3+}$ ions and its resonance ${ }^{47}$. It should be noted that the oscillator strength of $\mathrm{Ti}^{4+}$ does not have such kind of feature. Most importantly, with the increase of $\mathrm{O}_{\mathrm{v}}$, the peak of the resonance states around $2 \mathrm{eV}$ gradually becomes much more apparent (Fig. 4e, f). Since the gap states have a direct relationship with the $\mathrm{O}_{\mathrm{v}}$ defects, the gap states can be greatly broadened and even extended to the $\mathrm{CBM}^{48}$. As a result, the energy difference between gap states and resonance states in $\mathrm{CB}$ dramatically decreases with the increase of $\mathrm{O}_{v}$, which falls into the energy range of the visible light region. This is why reduced $\mathrm{TiO}_{2}$ could absorb visible light and its apparent color changes from blue to dark with the increase of $\mathrm{O}_{\mathrm{v}}$ concentration.

\section{Discussion}

From the above results, it can be clearly seen that the lithiumreduced $\mathrm{TiO}_{2}$ nanoparticles show significantly enhanced photocatalytic properties compared with pristine $\mathrm{TiO}_{2}$, which may be correlated with the greatly enhanced light absorption, improved conductivity, surface disorder layer, implanted oxygen vacancies, and generated $\mathrm{Ti}^{3+}$. In particular, the improved light absorption and photocatalytic properties are attributed to the $\mathrm{Ti}^{3+}$ of the defective $\mathrm{TiO}_{2}$. Furthermore, metallic conduction can be achieved at the crystalline-amorphous interface of the defective $\mathrm{TiO}_{2}$ nanoparticles, which enhances the electronic transport properties of $\mathrm{TiO}_{2}{ }^{34}$. Another important factor is the implanted oxygen vacancies and/or surface disorder, which also plays a key role in increasing the photocatalytic activity. The donor density of $\mathrm{TiO}_{2}$ is enhanced by introducing oxygen vacancies since they can act as 
electron donors ${ }^{49}$, which improves charge transport and shifts the Fermi level towards the conduction band ${ }^{50}$, facilitating charge separation and improving the incident photon-to-current efficiency (IPCE) in the UV region ${ }^{51}$. Finally, the generated $\mathrm{Ti}^{3+}$ in the defective $\mathrm{TiO}_{2}$ can reduce the recombination of the photogenerated electron-hole pairs and thus improve the photocatalytic activity of the lithium-reduced $\mathrm{TiO}_{2}$ nanoparticles ${ }^{19}$.

We note that the contact reaction between the $\mathrm{Li}$ and $\mathrm{TiO}_{2}$ powders proceeds very quickly in principle, since Li can diffuse quickly in the oxides. However, we observed from Supplementary Fig. 1 that the color of the mixed powders turned darker after grinding for longer times, indicating that the defects formation requires long reaction times. This is because lithium oxide passivation layers (like $\mathrm{Li}_{2} \mathrm{O}$ ), formed at the interface between $\mathrm{Li}$ and defective oxide powders, buffer the reaction between $\mathrm{Li}$ and the oxides. Therefore, only a small amount of Li diffuses into the oxides at the Li/oxide interface. However, with continuous handgrinding, the oxide passivation layer was destroyed by the shearing and friction forces between particles, with fresh lithium exposed to react with fresh oxide surfaces. After lengthy grinding process, the lithium oxide passivation layer was cyclically formed and destroyed, and the lithium finally reacted entirely with oxide powders, resulting in the formation of uniform defective oxide powders. While we used hand-grinding here to demonstrate the successful Li-reduction and defect-implanting chemistry, ballmilling or machine grinding could also be introduced to speed up the reactions, making the process more controllable and timeefficient in the future study. Thus, we suggest that almost all of our lithium-reduced oxide nano-powders have a defective structure based on the following reasons: (1) Besides TEM, the defect structures have also been confirmed by XPS spectra and EPR spectra (Fig. 2 and Supplementary Fig. 10), which provided the average overall analysis of the materials. (2) The defective oxide powders have demonstrated significantly enhanced photocatalytic properties after lithium reduction. In addition, the materials undergo uniform color changes from white to black. (3) Our experimental procedure ensures that all substrates are evenly involved in the reaction. Firstly, we chose oxide nanoparticles and $\mathrm{Li}$ powders instead of micro oxide powders and $\mathrm{Li}$ foil as the substrates to avoid possible uneven contact. Secondly, we added solvent (DMC) to enhance their contact while grinding. Lastly, we applied long-time $(\sim 1 \mathrm{~h})$ grinding process to allow the oxide particles to evenly attach and react with the lithium powder. (4) Our experimental and theoretical discussion also pointed out that the $\mathrm{Li}$ atoms have a high reaction rate with oxides; that is to say, the defect implanting reaction is fast, which further assists the formation of uniform defects in the nanoparticles.

\section{Methods}

Materials preparation. The used raw materials are commercially available; they are $\mathrm{TiO}_{2}$ (99.9\%, Degussa), $\mathrm{ZnO}$ (99.9\%, Aladdin), $\mathrm{SnO}_{2}$ (99.9\%, Aladdin), $\mathrm{CeO}_{2}$ (99.5\%, Aladdin), and Li powders (99.9\%, Cellithium, China). A certain amount of oxide material was weighed and put in a mortar, then lithium powders and DMC (99\%, Aladdin) were added acting as reducing agent and dispersant, respectively. In order to protect the lithium powders from oxidizing by air, the milling process was conducted in a glove box filled with Ar. The materials were carefully mixed by hand, grinding with a speed of $\sim 2$ laps per second for $1 \mathrm{~h}$ and removed to dissolve the generated lithium oxide by dilute $\mathrm{HCl}$. After centrifuging and washing by deionized water three times, the obtained oxide powders were dried for characterization. In this study, different contents of lithium metal powder were added to the oxide nanoparticles to engineer the defect content. It is noted that although highly active Li metal powders were used in the experiment, there is minimal danger in the whole experimental process during regular operation. Firstly, the lithium powders used in this work are mass-produced in industry and treated by surface passivation. Furthermore, the lithium powders were put into a sealed glove box full of argon for safe storage and usage. Lastly, only a small amount of lithium powder (less than $5 \mathrm{wt} \%$ ) is required by our method.
Structural characterization. The crystal structures were investigated by X-ray diffraction (XRD, D/max-2500, Rigaku). The morphology and microstructure were analyzed by SEM (MERLIN VP Compact, Zeiss) and aberration-corrected highresolution transmission electron microscopy (JEM-ARM200F, JEOL). The X-ray photoelectron spectra of the $\mathrm{TiO}_{2}$ samples were measured at beamline $\mathrm{BL} 10 \mathrm{~B}$ of Hefei National Synchrotron Radiation Laboratory (NSRL) in China. The binding energies of $\mathrm{ZnO}, \mathrm{SnO}_{2}$, and $\mathrm{CeO}_{2}$ were characterized using an $\mathrm{X}$-ray photoelectron spectrometer (XPS, Escalab 250Xi, Thermo Fisher Scientific). The absorption spectra were collected by UV-Vis-NIR spectrophotometer (UV-2600, Shimadzu). The defects were detected by EPR spectroscopy (FA-200, JEOL). The Raman spectra were measured on a microscopic confocal Raman spectrometer (Raman, LabRAM HR800, HORIBA Jobin Yvon) using a $532 \mathrm{~nm}$ laser as the excitation source. The specific surface area was measured by a surface area analyzer (QuadraSorb SI, Quantachrome). The solid steady-state fluorescence and transient state fluorescence spectra were tested by fluorescence spectrometer (FLS920, Edinburgh Instruments). The impedance spectra were measured by an electrochemical workstation (PGSTAT204, Autolab) with a three-electrode system in 0.5 $\mathrm{M} \mathrm{H}_{2} \mathrm{SO}_{4}$ solution, in which $\mathrm{Ag} / \mathrm{AgCl}$ electrode and carbon rod were used as reference electrode and counter electrode. The $\mathrm{TiO}_{2}$ nanoparticles were dispersed ultrasonically in deionized water and ethanol (volume ratio $=1: 1$ ) mixed solution with Nafion 117 (Aldrich) as binder for $30 \mathrm{~min}$ and then dipped on carbon fiber paper (Toray) with a loading of $0.1 \mathrm{mg} \mathrm{cm}^{-2}$ as working electrode.

Photocatalytic measurements. The photocatalytic activity was performed under simulated solar light (PLS-SXE 300C, Perfectlight) with AM1.5G at a light density of $100 \mathrm{~mW} \mathrm{~cm}^{-2}$. The solution with $\mathrm{RhB}\left(2.5 \times 10^{-5} \mathrm{~mol} \mathrm{~L}^{-1}\right)$ and $\mathrm{TiO}_{2}$ nanoparticles $\left(1 \mathrm{mg} \mathrm{ml}^{-1}\right)$ was ultrasonicated for $30 \mathrm{~min}$, magnetically stirred for $1 \mathrm{~h} \mathrm{in}$ the dark and then irradiated under a full solar light. The concentration of RhB was measured by a spectrophotometer (UV-2600, Shimadzu). In the photocatalytic degradation cycle experiments, the $\mathrm{TiO}_{2}$ nanoparticles were centrifuged, washed and dried for the next cycle, and the process was repeated nine times. In the photocatalytic hydrogen evolution experiments, Pt nanoparticles were applied as a co-catalyst by adding chloroplatinic acid in the solution with $\mathrm{TiO}_{2}$ nanoparticles under UV light for $1 \mathrm{~h}$. Methanol $(10 \mathrm{ml}), 40 \mathrm{ml}$ deionized water, and $1.25 \mathrm{mg}$ $\mathrm{TiO}_{2}$ nanoparticles $(1 \% \mathrm{Pt})$ were added in a closed glass beaker. The solution was irradiated by a full solar light under $\mathrm{Ar}$ atmosphere and the generated $\mathrm{H}_{2}$ was determined by a gas chromatographer (GC-7920, CEAULIGHT). For the stability experiments, the same solution was continually irradiated for 11 cycles. Before each run, extra methanol was added to make the volume of the solution reach to $50 \mathrm{ml}$.

Calculation details. First-principles calculations were performed with the $\mathrm{CP} 2 \mathrm{~K} /$ Quickstep package, in which a hybrid Gaussian and plane-waves was applied ${ }^{52}$. In such method, the valence electrons were expanded in terms of Gaussian functions with molecularly optimized double- $\zeta$ polarized basis sets $(m-D Z V P)^{53}$. For the auxiliary basis set of plane waves, a 320 Ry cut-off was used. The generalized gradient approximation of Perdew, Burke, and Ernzerhof exchange-correlation functional was also chosen for calculation $s^{54}$. Hubbard U $(3.5 \mathrm{eV})$ correction was used to calculate the strong exchange interaction of Ti $3 d$ orbital as well as $\mathrm{O} 2 p$ orbita $^{55-57}$. Core electrons were described with norm-conserving Goedecker, Teter, and Hutter (GTH) pseudopotentials ${ }^{58}$. All atoms in the slab were relaxed until the maximum residual force is less than $0.02 \mathrm{eV}^{-1}$. To estimate the difficulty of $\mathrm{O}_{\mathrm{v}}$ diffusion from surface to subsurface, the transition states along the reaction pathways are searched by the Climbing Image Nudged Elastic Band (CINEB) approach ${ }^{59}$.

In order to know whether lithium can oxidize $\mathrm{TiO}_{2}, \mathrm{SnO}_{2}, \mathrm{ZnO}$ etc. as represented in Eq. (2), the reaction enthalpy was calculated. Enthalpy $H$ equals the internal energy $U$ plus pressure-volume work $\mathrm{pV}$, that is, $H=U+\mathrm{pV}$. Because all the energies are calculated in the solid state, the pressure-volume effect can be neglected. The reaction enthalpy is contributed mainly by the internal energy of the reactants or products. Here, the enthalpy change $\Delta H$ is calculated using the following formula:

$$
\Delta H(\mathrm{Li})=E_{\mathrm{MO}-\mathrm{O}_{\mathrm{v}}}+2 * E_{\mathrm{Li}}-E_{\mathrm{MO}}-E_{\mathrm{Li2O}},
$$

where $E_{\mathrm{MO}-\mathrm{O}_{\mathrm{v}}}, E_{\mathrm{Li}}, E_{\mathrm{MO}}$, and $E_{\mathrm{Li2}}$, are the total energy of bulk metal-oxide with $\mathrm{O}_{\mathrm{v}}$, total energy of a lithium atom in bulk phase, total energy of perfect bulk metaloxide and total energy of a lithium oxide unit in bulk phase, respectively. The formation energy $E_{\text {form }}$ is calculated by $E_{\text {form }}=E_{\mathrm{D}}-E_{\mathrm{P}}+0.5^{*} E_{\mathrm{O} 2}$, where $E_{\mathrm{D}}, E_{\mathrm{P}}$ and $E_{\mathrm{O} 2}$ are the total energy of defect slab, perfect slab and $\mathrm{O}_{2}$, respectively.

The anatase- $\mathrm{TiO}_{2}(110)$ surface was modeled using a repeated slab geometry with four $\mathrm{TiO}_{2}$ tri-layers and a $(1 \times 4)(10.35 \AA \times 15.21 \AA)$ surface supercell. The vacuum separation between slabs was around $15 \AA . \mathrm{O}_{\mathrm{v}}$ defects with different concentrations in the reduced anatase were modeled by removing one, two or four oxygen atoms from the subsurface of the supercell used in this work; see Supplementary Fig. 16. Due to the large computational resources for CI-NEB, a three tri-layers slab was adopted for the estimation of $\mathrm{O}_{\mathrm{v}}$ transition.

The oscillator strength was calculated using the following equation ${ }^{60}$ :

$$
f_{c v}^{\mu}=\frac{2}{m_{e}\left(E_{c}-E_{v}\right)}\left|\left\langle v\left|p_{\mu}\right| c\right\rangle\right|^{2}
$$


where $f_{c v}^{\mu}$ is the oscillator strength in the $\vec{e}_{\mu}$ polarized direction. $\langle v|$ denotes Kohn -Sham orbitals corresponding to VBM state or gap state and $|c\rangle$ denotes Kohn -Sham orbitals corresponding to unoccupied MOs above $E_{\mathrm{F} .} E_{c}$ and $E_{v}$ correspond to the eigenvalue of $|c\rangle$ and $\langle v|$ 's orbital, respectively. $p_{\mu}$ is the momentum along $\vec{e}_{\mu}$ direction.

Data availability. The datasets generated during the current study are available from the corresponding author on reasonable request.

Received: 22 June 2017 Accepted: 9 March 2018

Published online: 03 April 2018

\section{References}

1. Jia, J. et al. Heterogeneous catalytic hydrogenation of $\mathrm{CO}_{2}$ by metal oxides: defect engineering-perfecting imperfection. Chem. Soc. Rev. 46, 4631-4644 (2017).

2. Kim, H. S. et al. Oxygen vacancies enhance pseudocapacitive charge storage properties of $\mathrm{MoO}_{3-\mathrm{x}}$. Nat. Mater. 16, 454-460 (2017).

3. Li, B. Q. et al. Regulating p-block metals in perovskite nanodots for efficient electrocatalytic water oxidation. Nat. Commun. 8, 934 (2017).

4. Chen, W. et al. Efficient and stable large-area perovskite solar cells with inorganic charge extraction layers. Science 350, 944-948 (2015).

5. Koketsu, T. et al. Reversible magnesium and aluminium ions insertion in cation-deficient anatase $\mathrm{TiO}_{2}$. Nat. Mater. 16, 1142-1148 (2017).

6. Das, S. et al. Controlled manipulation of oxygen vacancies using nanoscale flexoelectricity. Nat. Commun. 8, 615 (2017).

7. Giordano, F. et al. Enhanced electronic properties in mesoporous $\mathrm{TiO}_{2}$ via lithium doping for high-efficiency perovskite solar cells. Nat. Commun. 7, 10379 (2016).

8. Xu, Y. F. et al. Pd-catalyzed instant hydrogenation of $\mathrm{TiO}_{2}$ with enhanced photocatalytic performance. Energy Environ. Sci. 9, 2410-2417 (2016).

9. Sun, Q. B. et al. The formation of defect-pairs for highly efficient visible-light catalysts. Adv. Mater. 29, 1605123 (2017).

10. Wahlstrom, E. et al. Electron transfer-induced dynamics of oxygen molecules on the $\mathrm{TiO}_{2}(110)$ surface. Science 303, 511-513 (2004).

11. Lawrence, N. J. et al. Defect engineering in cubic cerium oxide nanostructures for catalytic oxidation. Nano Lett. 11, 2666-2671 (2011).

12. Li, M. F. et al. Ultrafine jagged platinum nanowires enable ultrahigh mass activity for the oxygen reduction reaction. Science 354, 1414-1419 (2016).

13. Runnerstrom, E. L. et al. Defect engineering in plasmonic metal oxide nanocrystals. Nano Lett. 16, 3390-3398 (2016).

14. Zhang, N. et al. Oxide defect engineering enables to couple solar energy into oxygen activation. J. Am. Chem. Soc. 138, 8928-8935 (2016).

15. Ou, G. et al. Arc-melting to narrow the bandgap of oxide semiconductors. Adv. Mater. 27, 2589-2594 (2015).

16. Liao, L. B. et al. Efficient solar water-splitting using a nanocrystalline $\mathrm{CoO}$ photocatalyst. Nat. Nanotechnol. 9, 69-73 (2014).

17. Chen, X. B., Liu, L. \& Huang, F. Q. Black titanium dioxide $\left(\mathrm{TiO}_{2}\right)$ nanomaterials. Chem. Soc. Rev. 44, 1861-1885 (2015).

18. Chen, X. B., Liu, L., Yu, P. Y. \& Mao, S. S. Increasing solar absorption for photocatalysis with black hydrogenated titanium dioxide nanocrystals. Science 331, 746-750 (2011).

19. Sinhamahapatra, A., Jeon, J. P. \& Yu, J. S. A new approach to prepare highly active and stable black titania for visible light-assisted hydrogen production. Energy Environ. Sci. 8, 3539-3544 (2015).

20. Wang, Z. et al. Visible-light photocatalytic, solar thermal and photoelectrochemical properties of aluminium-reduced black titania. Energy Environ. Sci. 6, 3007-3014 (2013).

21. Nakajima, T., Tsuchiya, T. \& Kumagai, T. Pulsed laser-induced oxygen deficiency at $\mathrm{TiO}_{2}$ surface: anomalous structure and electrical transport properties. J. Solid State Chem. 182, 2560-2565 (2009).

22. Nakajima, T., Nakamura, T., Shinoda, K. \& Tsuchiya, T. Rapid formation of black titania photoanodes: pulsed laser-induced oxygen release and enhanced solar water splitting efficiency. J. Mater. Chem. A 2, 6762-6771 (2014).

23. Tan, H. et al. Efficient and stable solution-processed planar perovskite solar cells via contact passivation. Science 355, 722-726 (2017).

24. Zhang, $X$. et al. Direction-specific van der Waals attraction between rutile $\mathrm{TiO}_{2}$ nanocrystals. Science 356, 434-437 (2017).

25. Yang, W. S. et al. Iodide management in formamidinium-lead-halide-based perovskite layers for efficient solar cells. Science 356, 1376 (2017)

26. Narvaez, J., Vasquez-Sancho, F. \& Catalan, G. Enhanced flexoelectric-like response in oxide semiconductors. Nature 538, 219-221 (2016).

27. Vignes, A. Metallurgical Thermochemistry in Extractive Metallurgy 1: Basic Thermodynamics and Kinetics (John Wiley \& Sons, Inc., USA, 2013).

28. Fujishima, A. \& Honda, K. Electrochemical photolysis of water at a semiconductor electrode. Nature 238, 37-38 (1972).
29. Jing, L. Q., Zhou, W., Tian, G. H. \& Fu, H. G. Surface tuning for oxide-based nanomaterials as efficient photocatalysts. Chem. Soc. Rev. 42, 9509-9549 (2013).

30. $\mathrm{Hu}, \mathrm{W}$. B. et al. Electron-pinned defect-dipoles for high-performance colossal permittivity materials. Nat. Mater. 12, 821-826 (2013).

31. Lin, T. Q. et al. Effective nonmetal incorporation in black titania with enhanced solar energy utilization. Energy Environ. Sci. 7, 967-972 (2014).

32. Thorpe, R., Rangan, S., Sina, M., Cosandey, F. \& Bartynski, R. A. Conversion reaction of $\mathrm{CoO}$ polycrystalline thin films exposed to atomic lithium. J. Phys. Chem. C 117, 14518-14525 (2013).

33. Tang, K. C. et al. Distinguishing oxygen vacancy electromigration and conductive filament formation in $\mathrm{TiO}_{2}$ resistance switching using liquid electrolyte contacts. Nano. Lett. 17, 4390-4399 (2017).

34. Lu, X. J. et al. Conducting interface in oxide homojunction: understanding of superior properties in black $\mathrm{TiO}_{2}$. Nano Lett. 16, 5751-5755 (2016).

35. Amano, F., Nakata, M., Yamamoto, A. \& Tanaka, T. Effect of $\mathrm{Ti}^{3+}$ ions and conduction band electrons on photocatalytic and photoelectrochemical activity of rutile titania for water oxidation. J. Phys. Chem. C 120, 6467-6474 (2016).

36. Deskins, N. A. \& Dupuis, M. Electron transport via polaron hopping in bulk $\mathrm{TiO}_{2}$ : a density functional theory characterization. Phys. Rev. B 75, 19521219 (2007).

37. Kowalski, P. M., Camellone, M. F., Nair, N. N., Meyer, B. \& Marx, D. Charge localization dynamics induced by oxygen vacancies on the titania $\mathrm{TiO}_{2}(110)$ surface. Phys. Rev. Lett. 105, 146405 (2010).

38. Spreafico, C. \& VandeVondele, J. The nature of excess electrons in anatase and rutile from hybrid DFT and RPA. Phys. Chem. Chem. Phys. 16, 26144-26152 (2014).

39. Cheng, H. Z. \& Selloni, A. Energetics and diffusion of intrinsic surface and subsurface defects on anatase $\mathrm{TiO}_{2}(101)$. J. Chem. Phys. 131, 54703 (2009).

40. He, Y. B., Dulub, O., Cheng, H. Z., Selloni, A. \& Diebold, U. Evidence for the predominance of subsurface defects on reduced anatase $\mathrm{TiO}_{2}(101)$. Phys. Rev. Lett. 102, 10610510 (2009).

41. Cheng, H. Z. \& Selloni, A. Surface and subsurface oxygen vacancies in anatase TiO2 and differences with rutile. Phys. Rev. B 79, 0921019 (2009).

42. Khan, Z., Khannam, M., Vinothkumar, N., De, M. \& Qureshi, M. Hierarchical 3D NiO-CdS heteroarchitecture for efficient visible light photocatalytic hydrogen generation. J. Mater. Chem. 22, 12090-12095 (2012).

43. $\mathrm{Hu}, \mathrm{Y}$. H. A highly efficient photocatalyst hydrogenated black $\mathrm{TiO}_{2}$ for the photocatalytic splitting of water. Angew. Chem. Int. Ed. 51, 12410-12412 (2012).

44. Yim, C. M., Pang, C. L. \& Thornton, G. Oxygen vacancy origin of the surface band-gap state of $\mathrm{TiO}_{2}(110)$. Phys. Rev. Lett. 104, 36806 (2010).

45. Mao, X. C. et al. Band-gap states of $\mathrm{TiO}_{2}(110)$ : major contribution from surface defects. J. Phys. Chem. Lett. 4, 3839-3844 (2013).

46. Wang, Z. Q. et al. Localized excitation of $\mathrm{Ti}^{3+}$ ions in the photoabsorption and photocatalytic activity of reduced rutile $\mathrm{TiO}_{2}$. J. Am. Chem. Soc. 137, 9146-9152 (2015)

47. $\mathrm{Li}$, J. et al. Unraveling the origin of visible light capture by core-shell $\mathrm{TiO}_{2}$ nanotubes. Chem. Mater. 28, 4467-4475 (2016).

48. Henrich, V. E., Dresselhaus, G. \& Zeiger, H. J. Observation of 2-dimensional phases associated with defect states on surface of $\mathrm{TiO}_{2}$. Phys. Rev. Lett. 36, 1335-1339 (1976)

49. Janotti, A. et al. Hybrid functional studies of the oxygen vacancy in $\mathrm{TiO}_{2}$. Phys. Rev. B 81, 85212 (2010).

50. Cronemeyer, D. C. Infrared absorption of reduced rutile $\mathrm{TiO}_{2}$ single crystals. Phys. Rev. 113, 1222-1226 (1959).

51. Wang, G. M. et al. Hydrogen-treated $\mathrm{TiO}_{2}$ nanowire arrays for photoelectrochemical water splitting. Nano Lett. 11, 3026-3033 (2011).

52. VandeVondele, J. et al. QUICKSTEP: fast and accurate density functional calculations using a mixed Gaussian and plane waves approach. Comput. Phys. Commun. 167, 103-128 (2005).

53. VandeVondele, J. \& Hutter, J. Gaussian basis sets for accurate calculations on molecular systems in gas and condensed phases. J. Chem. Phys. 127, 114105 (2007).

54. Perdew, J. P., Burke, K. \& Ernzerhof, M. Generalized gradient approximation made simple. Phys. Rev. Lett. 77, 3865-3868 (1996).

55. Liechtenstein, A. I., Anisimov, V. I. \& Zaanen, J. Density-functional theory and strong-interactions-orbital ordering in Mott-Hubbard insulators. Phys. Rev. B 52, R5467-R5470 (1995).

56. Schuler, M., Rosner, M., Wehling, T. O., Lichtenstein, A. I. \& Katsnelson, M. I. Optimal hubbard models for materials with nonlocal coulomb interactions: graphene, silicene, and benzene. Phys. Rev. Lett. 111, 366013 (2013).

57. Ataei, S. S., Mohammadizadeh, M. R. \& Seriani, N. Ab initio simulation of the effects of hydrogen concentration on anatase $\mathrm{TiO}_{2}$. J. Phys. Chem. C 120, 8421-8427 (2016).

58. Goedecker, S., Teter, M. \& Hutter, J. Separable dual-space Gaussian pseudopotentials. Phys. Rev. B 54, 1703-1710 (1996). 
59. Henkelman, G. \& Jónsson, H. Improved tangent estimate in the nudged elastic band method for finding minimum energy paths and saddle points. J. Chem. Phys. 113, 9978-9985 (2000).

60. Pan, D., Wan, Q. \& Galli, G. The refractive index and electronic gap of water and ice increase with increasing pressure. Nat. Commun. 5, 3919 (2014).

\section{Acknowledgements}

This work was supported by the National Basic Research of China (Grants 2015CB932500), National Natural Science Foundations of China (Grant 51661135025 51522207, 51572016, and U1530401). G.O. and K.H. acknowledge the support from Project funded by China Postdoctoral Science Foundation (2016M600079 and 2016M601019). We also thank Prof. H.X. Ju and Mr. S.W. Hu (Beamline BL10B of Hefei National Synchrotron Radiation Laboratory (NSRL) in China) for their great help in the XPS experiment. This research is supported by a Tianhe-2JK computing time award at the Beijing Computational Science Research Center (CSRC).

\section{Author contributions}

G.O., Y.X., Y. Liang, and C.Y. fabricated the samples. G.O., Y.X., R.L., K.H., D.Z., and W. C. performed the measurements. B.G. and R.Y. performed the TEM results. B.W., Y.T., J. L. and L.-M.L. developed the theory. H.W. and Y. Li designed the project. All authors discussed the result, and contributed to the writing of the manuscript.

\section{Additional information}

Supplementary Information accompanies this paper at https://doi.org/10.1038/s41467018-03765-0
Competing interests: The authors declare no competing interests.

Reprints and permission information is available online at http://npg.nature.com/ reprintsandpermissions/

Publisher's note: Springer Nature remains neutral with regard to jurisdictional claims in published maps and institutional affiliations.

cc (i) Open Access This article is licensed under a Creative Commons Attribution 4.0 International License, which permits use, sharing, adaptation, distribution and reproduction in any medium or format, as long as you give appropriate credit to the original author(s) and the source, provide a link to the Creative Commons license, and indicate if changes were made. The images or other third party material in this article are included in the article's Creative Commons license, unles indicated otherwise in a credit line to the material. If material is not included in the article's Creative Commons license and your intended use is not permitted by statutory regulation or exceeds the permitted use, you will need to obtain permission directly from the copyright holder. To view a copy of this license, visit http://creativecommons.org/ licenses/by/4.0/.

(C) The Author(s) 2018 